\title{
Corela
}

Cognition, représentation, langage

HS-9 | 2010

Parole

\section{Emphase prosodique et emphase syntaxique : le cas de « do » dans un corpus de parole naturelle.}

\section{Sophie Herment}

\section{OpenEdition}

\section{Journals}

Édition électronique

URL : https://journals.openedition.org/corela/1059

DOI : $10.4000 /$ corela. 1059

ISSN : 1638-573X

Éditeur

Cercle linguistique du Centre et de l'Ouest - CerLICO

\section{Référence électronique}

Sophie Herment, «Emphase prosodique et emphase syntaxique : le cas de « do » dans un corpus de parole naturelle. », Corela [En ligne], HS-9 | 2010, mis en ligne le 17 janvier 2011, consulté le 18 juin 2021. URL : http://journals.openedition.org/corela/1059 ; DOI : https://doi.org/10.4000/corela.1059

Ce document a été généré automatiquement le 18 juin 2021

\section{(c) (i) (2) (2)}

Corela - cognition, représentation, langage est mis à disposition selon les termes de la licence Creative Commons Attribution - Pas d'Utilisation Commerciale - Partage dans les Mêmes Conditions 4.0 International. 


\title{
Emphase prosodique et emphase syntaxique : le cas de « do » dans un corpus de parole naturelle.
}

\author{
Sophie Herment
}

\section{Introduction}

1 L'emphase est un phénomène large, présent partout dans le discours et qui peut prendre différentes formes. C'est à l'emphase dans son sens large, à savoir la mise en relief, d'une façon ou d'une autre d'un ou plusieurs éléments d'un énoncé, que nous nous sommes intéressée dans une large étude (Herment-Dujardin, 2001). Cette étude était dédiée aux corrélats acoustiques et prosodiques de l'emphase. Une fois ces critères définis, c'est sur les structures syntaxiques que nous souhaitons nous pencher désormais pour voir quels liens elles entretiennent avec la prosodie. C'est bien entendu les structures syntaxiques focalisantes qui vont attirer toute notre attention puisqu'elles mettent également en relief un ou plusieurs éléments d'un énoncé. Ce sont ainsi les relations étroites entre les deux types de mise en relief, prosodique et syntaxique, que nous allons tenter de mettre au jour. Plusieurs questions se posent en effet : comment la prosodie et la syntaxe se combinent-elles dans les mises en relief? L'une disparait-elle au profit de l'autre, ou bien l'une prend-elle simplement l'ascendant sur l'autre? Peut-on trouver une prosodie typique de certaines structures de focalisation?

2 Pour tenter d'apporter des éléments de réponse à ces questions, nous étudierons ici le cas de l'insertion de l'auxiliaire « do » en contexte affirmatif. Cette analyse sera basée sur un corpus de parole naturelle, dans lequel les énoncés sélectionnés seront analysés de façon acoustique et prosodique. 


\section{Corpus, méthode et définition}

\section{I.1. Le corpus de parole naturelle Aix-Marsec}

Nous avons utilisé pour la présente étude le corpus AIX-MARSEC (Auran et al., 2004) : c'est un corpus de parole naturelle, c'est-à-dire que les situations de communication sont naturelles. Il s'agit d'extraits de la $\mathrm{BBC}$, qui regroupent plusieurs styles de parole différents, allant des informations, des commentaires sportifs et de l'état de la route à des interviews ou des reportages en passant par des lectures de poésies ou de romans et des extraits de la messe. Il y a environ 5 heures et demi de parole. Ce corpus présente le gros avantage qu'il est déjà étiqueté, orthographiquement bien entendu, mais aussi phonétiquement et prosodiquement. C'est à cet étiquetage, réalisé à Aix-en-Provence au LPL (Laboratoire Parole et Langage) en grande partie par Caroline Bouzon et Cyril Auran, qu'il doit son nom d'Aix-Marsec. Il s'agissait au départ du corpus SEC (Spoken English Corpus) qui est devenu MARSEC (Machine Readable Spoken English Corpus) avec Peter Roach (Roach et al., 1993), puis Aix-Marsec au LPL.

L'étiquetage consiste en 8 niveaux que l'on voit ici sur la figure 1, qui représente la capture d'une fenêtre de PRAAT (Boersma \& Weenik, 1996), avec l'oscillogramme en haut, le spectrogramme en dessous sur lequel est apposée la courbe de fréquence fondamentale en bleu :

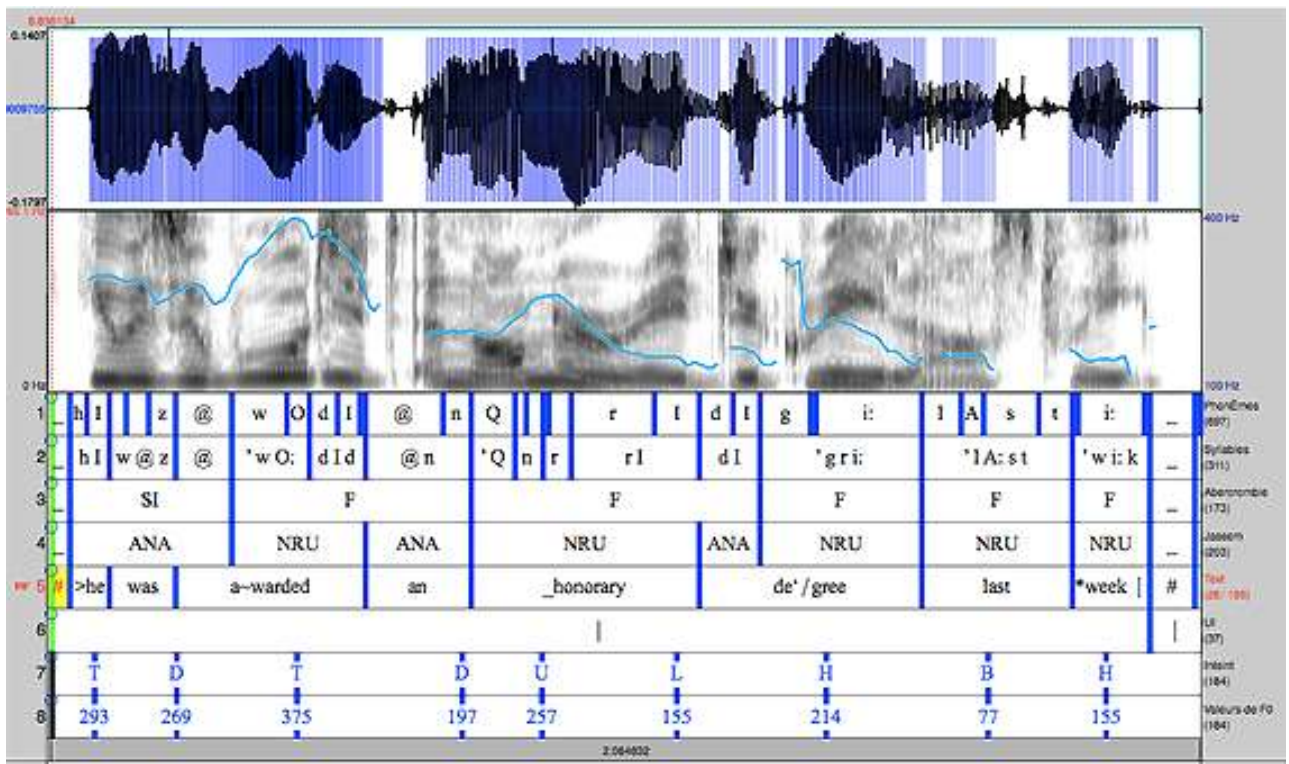

Figure 1 : Aix-Marsec

Les 8 niveaux (appelés « tiers » dans PRAAT) sous le spectrogramme sont :

-1. Les phonèmes (en alphabet SAMPA ${ }^{1}$ )

-2. Les syllabes

-3. Les pieds selon la théorie d'Abercrombie

-4. Les unités rythmiques selon la théorie de Jassem

-5. Le texte annoté des Tonetic Stress Marks (TSM, cf. I. 2.)

-6. Le découpage en unités intonatives (UI)

- 7. Le codage INTSINT (cf. I. 3.)

- 8. Les valeurs de F0 correspondant aux points-cible de INTSINT. 
6 Les 4 premiers niveaux n'ont pas été utilisés pour cette étude, mais les 4 suivants ont été exploités.

\section{2. Les « Tonetic Stress Marks »}

7 Le corpus SEC a été annoté prosodiquement par les phonéticiens G. Knowles et B. Williams. Les annotations ont ensuite été converties en 14 symboles ASCII (Roach, 1994) pour être facilement lues par un ordinateur. Elles sont montrées dans le tableau cidessous :

\begin{tabular}{|l|l|}
\hline- & low level \\
\hline$\sim$ & high level \\
\hline$<$ & step-down \\
\hline$>$ & step-up \\
\hline$/$ & (high) rise-fall \\
\hline$/$ & high fall-rise \\
\hline$\backslash$ & high fall \\
\hline$/$ & high rise \\
\hline, & low rise \\
\hline, & low fall \\
\hline,$\backslash$ & (low rise-fall - not used) \\
\hline$\backslash$, & low fall-rise \\
\hline$*$ & stressed but unaccented \\
\hline I & minor intonation unit boundary \\
\hline$\|$ & major intonation unit boundary \\
\hline
\end{tabular}

8 Cet étiquetage prosodique a bien sûr été précieux pour notre analyse comme nous le verrons plus tard, même si quelques difficultés ont parfois été rencontrées que nous le commenterons plus bas. 


\section{I.3. Codage INTSINT et valeurs de F0 (niveaux 7 et 8 )}

Le codage INTSINT, International Transcription System for INTonation, (Hirst \& Di Cristo, 1998) consiste en 8 points cibles :

- 2 points cible non relatifs, qui se réfèrent aux valeurs extrêmes de la tessiture du locuteur : la valeur la plus haute, Top (T) et la valeur la plus basse, Bottom (B) ;

- 1 point cible indiquant la valeur moyenne, $\operatorname{Mid}(\mathrm{M})$, ce point est également non relatif aux autres;

- 5 points cible, relatifs ceux-là, qui sont définis par rapport au point qui se trouve juste avant : plus haut « higher » $(\mathrm{H})$, plus bas « lower » (L), même valeur « same » $(\mathrm{S})$, descente

« Downstep » (D) et montée « upstep » (U).

10 Il est à noter que «Downstep » et "Upstep » indiquent un changement mélodique moindre que «Lower» et «Higher», et que «Mid» est utilisé en début d'unité intonative uniquement.

11 A chaque point-cible est associée une valeur de F0, calculée automatiquement.

12 Le codage INTSINT et les valeurs de F0 se sont révélées très utiles en complément des TSM, en particulier pour les chutes puisque c'est grâce à ces deux niveaux que nous avons déterminé s'il s'agissait de grandes chutes ou pas, même quand les TSM ne donnaient que des chutes normales. Nous reviendrons d'ailleurs sur les TSM car nous avons parfois trouvé des conflits entre le codage INTSINT et les TSM. C'est alors que les valeurs de F0 et la courbe de PRAAT jouent un rôle important. Par exemple quand la TSM est un «low fall » (petite chute) mais que le codage INTSINT donne un «Top » suivi d'un «Lower » ou même d'un « Bottom » sur le même mot et que les valeurs de F0 sont très écartées, c'est qu'il s'agit alors une grande chute et non d'une petite chute.

\section{4. Focalisation prosodique}

Rappelons que le but de cette étude est de chercher à voir si une prosodie marquée, comme dans le cas d'une focalisation prosodique, va être associée ou non à une structure syntaxique focalisante, «do » emphatique ici, ou si la structure syntaxique suffit à elle seule à la mise en relief. Mais qu'est-ce qu'une focalisation prosodique ? Ce que nous appelons focalisation uniquement prosodique est la mise en relief qui n'utilise aucun procédé syntaxique, mais uniquement des caractéristiques prosodiques (cf. Herment-Dujardin, 2001).

La mise en relief peut être effectuée grâce à la fréquence fondamentale, c'est-à-dire aux variations de hauteur mélodique avec des mouvements mélodiques plus amples ou une hauteur plus élevée.

On peut aussi jouer sur la durée segmentale, c'est-à-dire la durée des phonèmes, des consonnes et des voyelles. On allonge en général le mot que l'on veut mettre en relief et l'on raccourcit les mots qui l'entourent.

L'intensité peut également varier. Elle augmente souvent avec la hauteur mélodique.

L'attaque du mot emphatique peut être plus marquée : si le mot commence par une plosive $(/ \mathrm{p} /, / \mathrm{t} /, / \mathrm{k} /, / \mathrm{b} /, / \mathrm{d} /, / \mathrm{g} /)$, la tenue de la plosion sera plus longue, on en verra un exemple plus loin, ou bien un coup de glotte peut précéder la voyelle initiale, ou encore la consonne initiale peut être allongée par exemple). 
19 Ces caractéristiques prosodiques vont-elles se retrouver lorsque la syntaxe est déjà marquée ? Etudions le cas du « do » emphatique.

\section{Focalisation syntaxique : le « do » emphatique.}

20 Ce que l'on appelle communément le « do » emphatique est ce « do » auxiliaire que l'on insère dans la phrase, en contexte affirmatif, alors qu'il n'est pas nécessaire au niveau de la syntaxe : «I do know », par exemple, dans le sens de « je sais bien » ou encore, « si je sais ». L'ébauche de l'étude du «do» emphatique sur un autre corpus (HermentDujardin, 2008) avait été entreprise, mais sur un nombre trop faible d'occurrences. Il est en effet assez difficile de trouver des occurrences en discours spontané. Nous avons donc voulu vérifier nos hypothèses sur le corpus AIX-MARSEC, dans lequel 21 occurrences (sur les $5 \mathrm{~h}$ et demi de parole) ont été relevées.

21 L'étude précédente portait sur la prédication et en effet, il est reconnu que le « do" communément appelé emphatique indique une prise de position sur la réalité du lien Sujet/Prédicat, que celle-ci soit niée ou renforcée. Nous nous étions alors demandée s'il y avait une prosodie différente entre ces deux prises de position. Nous reprendrons ici ces deux cas et verrons si les résultats coïncident.

Et puis certains auteurs, comme Lapaire \& Rotgé (1991) par exemple, précisent que «Do" auxiliaire en contexte affirmatif peut ne pas toujours être emphatique. Il appartient toujours à la catégorie du «do" qui ne nie pas le lien S/P mais qui en marque le simple assentiment ou l'attestation, ou encore il s'agit du « do » de déduction logique, de rappel ou d'inexorabilité ou bien d'actualisation effective. Dans ces cas, il est inaccentué. Cette catégorie de « do » est très intéressante, puisque l'on nous donne un indice prosodique ici en précisant que ce cas est inaccentué. C'est aussi une des rares grammaires qui donne des indices accentuels ou prosodiques.

23 En effet nous n'avons rien trouvé dans la littérature qui traitait de façon détaillée de la prosodie de « do » auxiliaire en contexte affirmatif.

24 Dans le corpus précédent (de parole spontanée), nous n'avions relevé aucune occurrence de «do » inaccentué. Dans Aix-Marsec, seules deux occurrences ont été trouvées. Alors qu'en est-il ? C'est le troisième cas de « do » que nous étudierons.

\section{1. Quand « do » nie la réalité du lien S/P}

Lorsque « do » remet en cause la prédication, la prosodie est très marquée, comme le montre la figure 2 ci-dessous, sur laquelle on peut voir la courbe de fréquence fondamentale en bleu, le texte annoté des TSM, le codage INTSINT et les valeurs de F0 (exemple sonore $\left.\mathrm{n}^{\circ} 1\right)$ : 


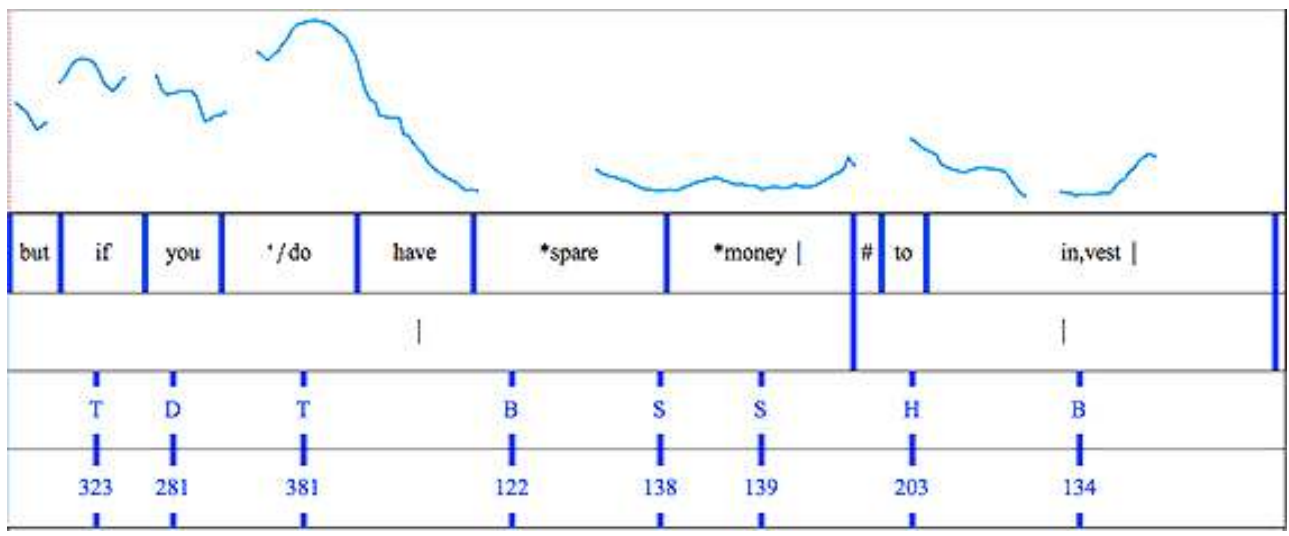

Figure 2: exemple sonore 1 note le «fall-rise » qui commence sur « do » avec une chute importante; «do » est le noyau et la remontée a lieu sur le dernier mot de l'unité intonative, «want» ici) et de surcroît la figure montre la tenue de la plosion du /d/ de " do ", en jaune ci-dessous, qui est de 0,119 s. Or nous avons mesuré d'autres /d/ chez cette même locutrice, toujours en début de syllabe accentuée ou en début de mot monosyllabique accentué au niveau de la phrase. Les résultats sont révélateurs puisqu'ils montrent que dans tous les autres cas, la tenue est bien moins longue : elle est de 0,06s sur le /d/ de «idea » par exemple, ou encore de $0,08 \mathrm{~s}$ sur un « did » en début de question et même de seulement $0,057 \mathrm{~s}$ sur une négation, un « don't ».

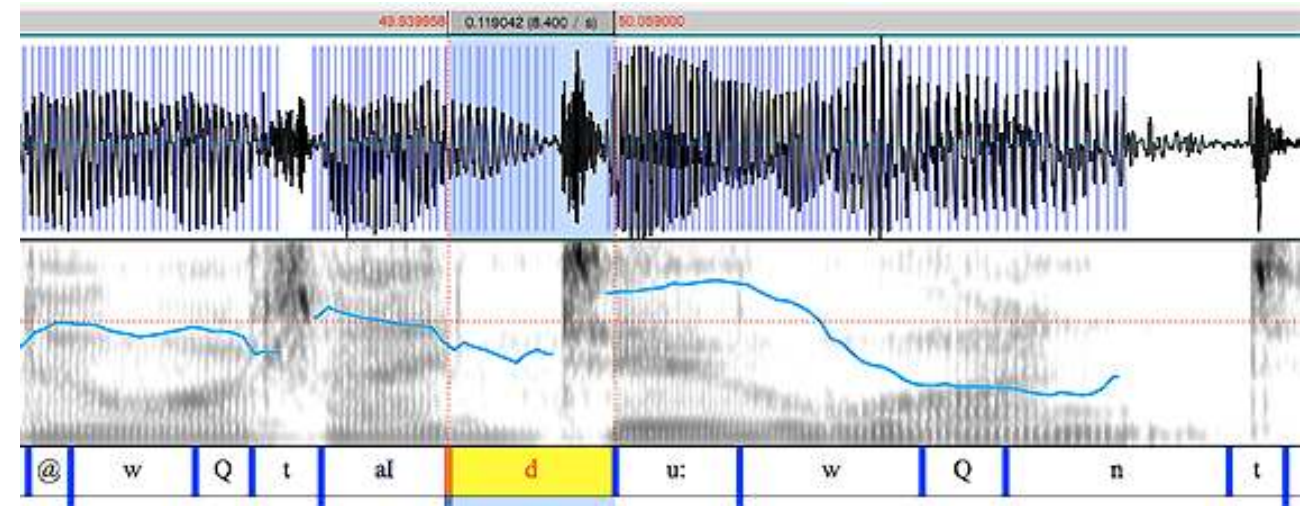

Figure 3: Exemple sonore $n^{\circ} 2$

D'autres exemples illustrent le même phénomène: accent nucléaire, mouvement mélodique ample et tenue plus importante de la plosion $\mathrm{du} / \mathrm{d} /:$ exemple sonore $\mathrm{n}^{\circ} 3$ : «...However, we do know that the Roman AS... ». 


\section{2. « Do » confirme le lien S/P}

Lorsque « do » a pour fonction de renforcer la prédication, et non plus de la nier, il est aussi porteur d'un accent, mais il ne s'agit plus de l'accent nucléaire cette fois, « do » est tonique de tête de l'unité intonative (c'est le premier accent de l'unité intonative qui est en général plus haut que le reste de l'UI ou bien plus rarement tout en bas dans le grave), et le mouvement mélodique est moins ample, de façon tout à fait logique, puisque les toniques de tête sont souvent des tons statiques aigus. La figure 4 , où seule est représentée la courbe de fréquence fondamentale, illustre ce phénomène. La sélection en bleu (morceau de courbe rouge) est le « did », «they did have trains » :

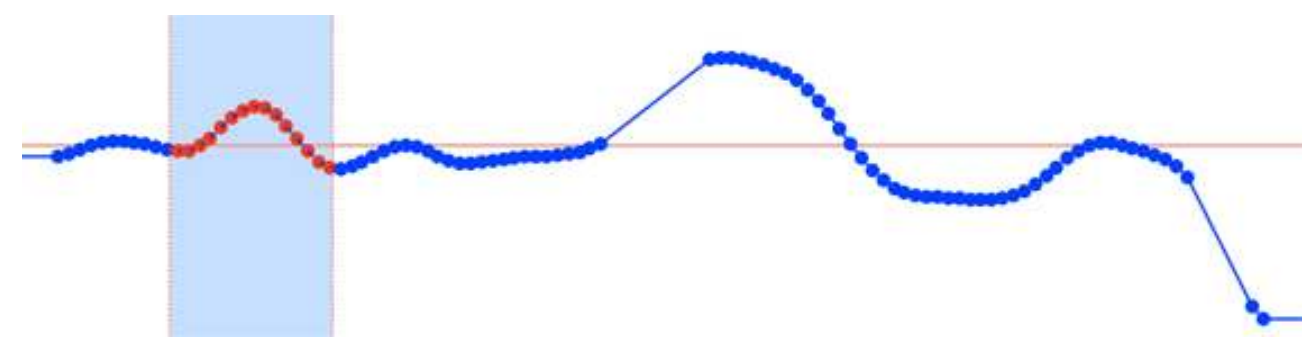

Figure 4: exemple sonore 4.

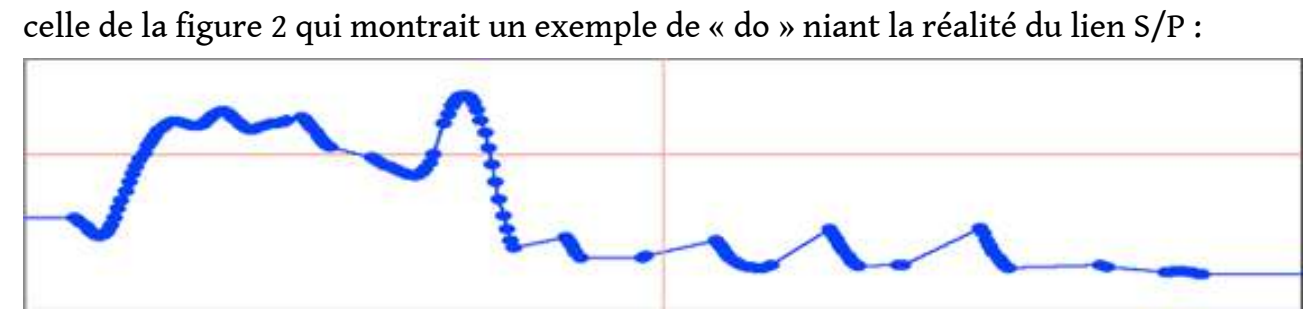

Dans cet exemple, «trains » est porteur de l'accent nucléaire (on entend bien le ton creusé, le «fall-rise » sur «trains » que l'on voit nettement sur la courbe et qui est typique d'un accent nucléaire) et non « did », qui est tonique de tête (head).

On peut entendre les mêmes caractéristiques prosodiques dans l'exemple sonore $\mathrm{n}^{\circ} 5$, «I say almost, because she did lose 3 times in 1986 »: « did » est tonique de tête, le noyau étant «lose» et sur lequel on entend que la locutrice fait un ton creusé également.

\section{3. Comparaison avec la focalisation uniquement prosodique :}

Comparons maintenant avec des focalisations uniquement prosodiques. Regardons une négation d'abord, puisqu'il s'agit en fait du même principe. La prédication est niée par la négation.

La courbe de F0 montrée dans la figure 5 (exemple sonore $\left.n^{\circ} 6\right)$ ressemble tout à fait à celle de la figure 2 qui montrait un exemple de « do » niant la réalité du lien S/P :

Figure 5: exemple sonore $n^{\circ} 6$

On voit que l'on retrouve exactement les mêmes structures prosodiques : un mouvement mélodique ample (une grande chute) sur «don't » ici, qui est également porteur de l'accent nucléaire (on le voit bien sur la courbe de F0 en bleu ci-dessus, 
puisqu'après la grande chute, le reste de l'unité intonative est désaccentué : dans le grave et relativement plat).

Il en est de même pour une focalisation prosodique sur autre chose qu'une négation, sur un mot de l'énoncé qui est mis en relief pour des raisons sémantiques par exemple, comme dans l'exemple sonore $n^{\circ} 7$ illustré figure 6 , où « violence » est très emphatique :

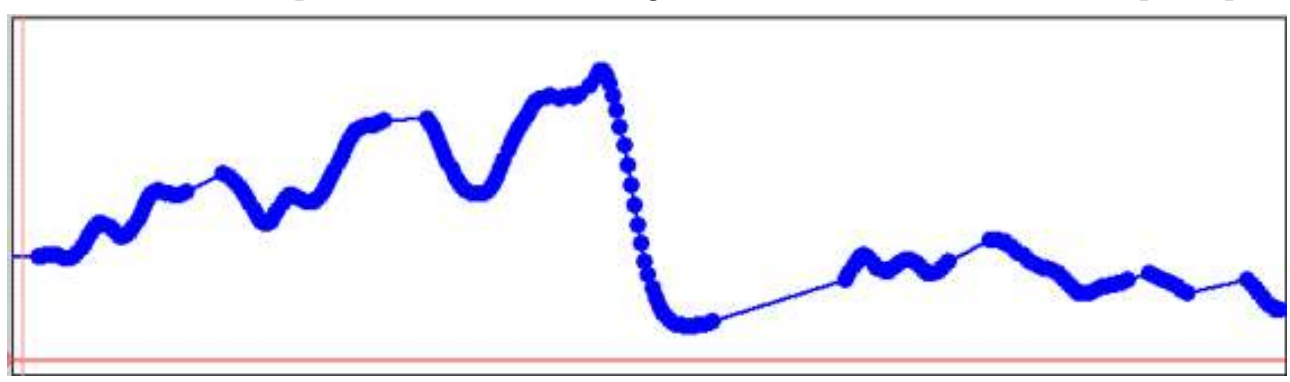

Figure 6: exemple sonore $n^{\circ} 7$ : «Or would you say domestic violence is a private issue ? »

La courbe de F0 est très similaire à la précédente (figure 5) et l'on retrouve en effet à nouveau exactement les mêmes caractéristiques prosodiques : l'accent nucléaire sur le mot mis en relief ("violence ») et un mouvement mélodique très ample (une grande chute ici).

Nous nous sommes ensuite intéressée au cas des auxiliaires qui renforcent la prédication, et l'on retrouve cette fois les mêmes caractéristiques prosodiques que pour le « do » emphatique qui renforce la prédication, c'est-à-dire un mouvement mélodique moins ample et l'auxiliaire sera tonique de tête et non pas noyau de l'unité intonative. L'exemple sonore $n^{\circ} 8$ (figure 7 ci-dessous) montre cela. La sélection est sur l'auxiliaire « is », « And it is theatre » : on pourrait traduire par « et il s'agit bien de théâtre »:

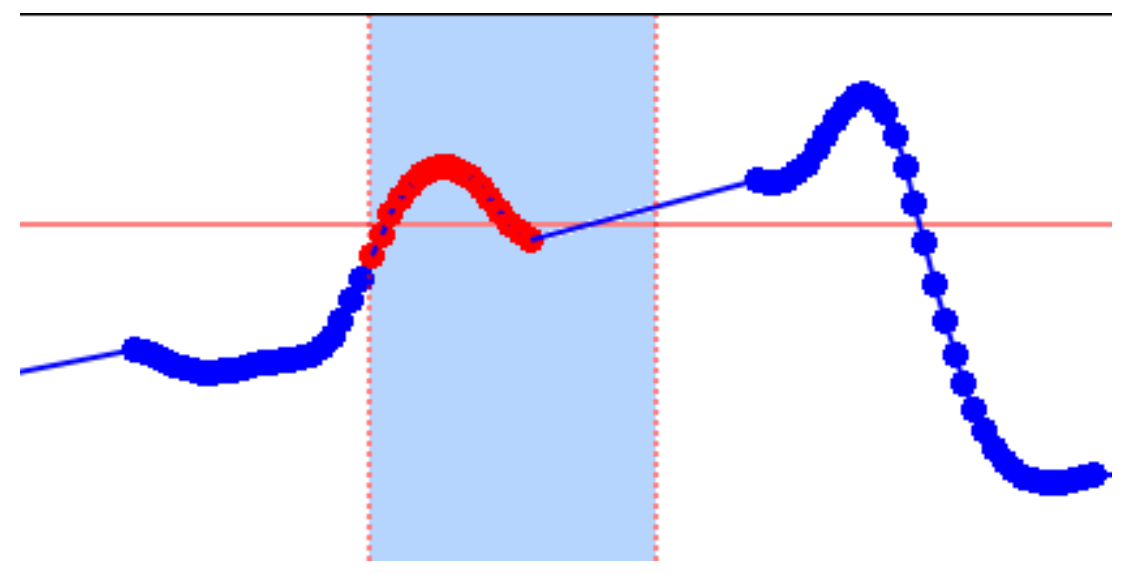

Figure 7 : exemple sonore $n^{\circ} 8:$ " and it is theatre »

Il est clair sur la figure 7 que « is » n'est pas noyau de l'unité intonative puisqu'on a une grande chute sur le mot suivant «theatre ", qui est le noyau, mais « is » est la tonique de tête : il est bien plus haut que le début de l'unité intonative).

L'exemple sonore $n^{\circ} 9$ avec l'auxiliaire «can » qui renforce le lien S/P présente encore les mêmes traits prosodiques: "Once you hear that you can actually communicate in their language ». «Can » est la tonique de tête et le noyau est « language ». 
Ainsi, il semblerait que le «do» communément appelé emphatique suive les mêmes « règles " prosodiques que les autres mots ayant les mêmes fonctions. Pourtant comme nous l'avons dit plus haut, "do" emphatique représente une importante marque syntaxique de focalisation puisqu'il n'est pas nécessaire au niveau de la syntaxe. Dans le cas du « do » emphatique, il semblerait donc que la focalisation syntaxique ne prenne pas la place de la focalisation prosodique, mais bien que les deux marques de focalisation soient complémentaires.

Nous avons pourtant mentionné plus haut que certaines grammaires répertorient le cas de certains «do » auxiliaires en contexte affirmatif qui seraient non accentués. Cela remet alors en question ce que nous venons de dire.

\section{4. « Do » inaccentué}

2 Comme nous l'avons expliqué en I.2., le corpus AIX-MARSEC est annoté grâce à des TSM et certains mots portent une étoile signifiant "stressed but unaccented" (ce qui signifie accentué en tant que mot mais pas accentué au niveau de la phrase).

3 Trois occurrences de « do » auxiliaire en contexte affirmatif portent cette TSM :

- Exemple sonore $\mathrm{n}^{\circ} 10$ : «And we certainly did have some dire matches in Mexico »

- Exemple sonore $n^{\circ} 11$ : «I really do think it's better to work for a voluntary organisation like VSO»

- Exemple sonore $n^{\circ} 12$ : It does seem especially unfair to the young "

Ce sont tous des cas où le lien S/P précédent est renforcé. Aucun n'est contrastif.

Nous avons indiqué en début d'exposé que nous n'avions trouvé que deux occurrences de ce «do » inaccentué : en effet la troisième occurrence (exemple sonore $n^{\circ} 12$ ) nous semble contestable. La courbe de fréquence fondamentale montrée figure 8 indique clairement que «does » (sélectionné sur la figure) est la tonique de tête de l'unité intonative puisqu'il est plus haut que tout le reste et est le premier élément de l'unité qui soit haut. Il ne présente pas de mouvement mélodique ample, c'est un statique aigu, comme c'est souvent le cas pour les toniques de tête. Le noyau de l'unité intonative est clairement « young », la courbe de F0 est très révélatrice.

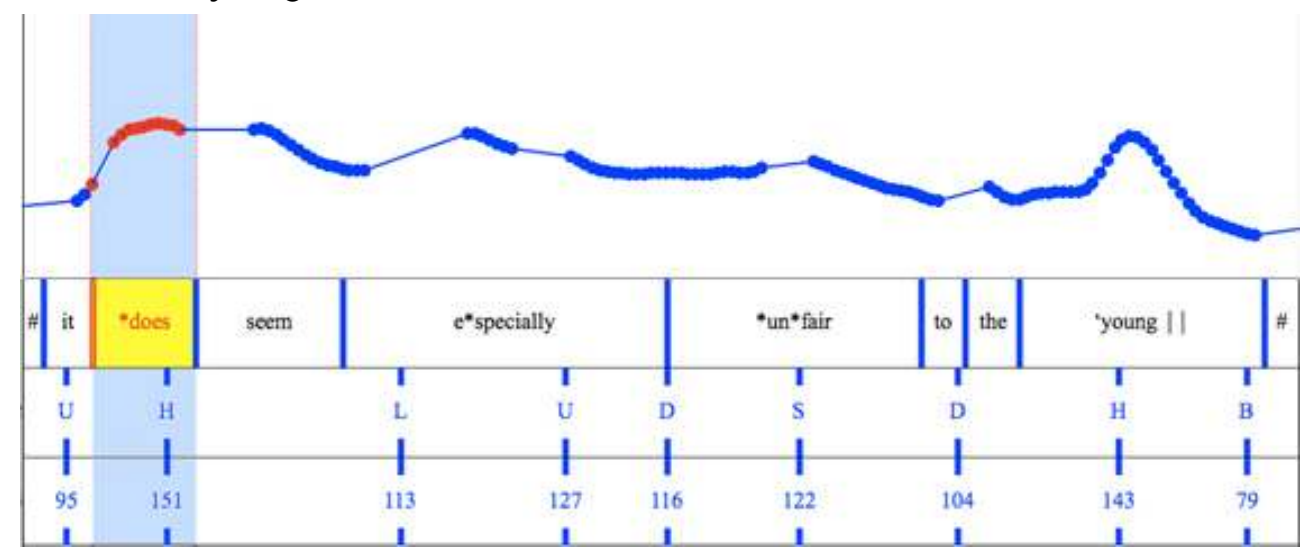

Figure 8 : exemple sonore $\mathrm{n}^{\circ} 12$

Cette analyse est confirmée par le codage INTSINT, qui indique un point cible $\mathrm{H}$ (Higher) sur «does ». Si « does » était «pre-head» (pré-tonique de tête), il serait au même niveau que l'élément précédent dans le médium probablement. Les valeurs de F0 
confirment également que « does » est bien accentué puisque $151 \mathrm{~Hz}$ est la valeur de F0 la plus haute de toute l'unité intonative.

Nous ne prendrons donc pas en compte cette occurrence dans les « do » inaccentués. Il s'agit d'un « do » qui renforce la relation prédicative et qui donc est tonique de tête et a les mêmes caractéristiques que les « do » décrits en II. 2.

Revenons aux deux premiers « do" de cette sous-section (exemples sonores 10 et 11). Ceux-ci semblent en effet bien être inaccentués. Tous deux ne nient pas la réalité du lien S/P et le premier (exemple sonore 10) appartient au cas que décrivent Lapaire et Rotgé (1991) (cf. II.) de simple assentiment ou d'acceptation: on pourrait gloser par "oui c'est vrai nous avons fait quelques mauvais matchs". Quant à la deuxième occurrence, il semble difficile de la caser dans la même catégorie. Elle semble correspondre aux cas de « do » qui renforcent la relation prédicative.

Alors pourquoi « do » n'est-il pas accentué ?

Il est intéressant de noter que dans les deux cas, un adverbe précède « do ", et c'est cet adverbe qui est porteur de l'accent. Il est tonique de tête dans le premier exemple, et noyau dans le deuxième. La réponse se trouve alors peut-être dans l'analyse de ces adverbes.

Peut-on se passer de « do » dans ces énoncés puisqu'il est inaccentué ? Dans le premier exemple, cela semble impossible à cause de la valeur de l'adverbe justement: "We certainly had some dire matches in Mexico » ne sonne pas bien (c'est l'entraîneur qui parle ici). Le fait d'avoir inséré « did » dans la syntaxe permet de donner à " certainly » sa valeur de " of course ", « without any doubt », alors que sans « did» il semble qu'il prendrait au contraire son sens de "there is some doubt left » et c'est la raison pour laquelle ça ne marcherait pas. Ainsi l'insertion de "did» ici semble être couplée à l'emploi de l'adverbe et lui donner sa valeur sémantique. L'adverbe étant en première position, c'est lui qui reçoit l'accent tonique de tête et «did» se retrouve désaccentué puisqu'il est couplé à l'adverbe.

Le deuxième exemple peut-il être traité de la même façon ? Il semble que non car ici on pourrait enlever le "do " sans problème et la phrase aurait le même sens. De plus l'adverbe ici est noyau de l'unité intonative, donc déjà fortement marqué prosodiquement et il s'agit d'un mot dont le sémantisme n'est pas neutre. Nous avons l'impression qu'ici «do» renforce non pas la relation prédicative, mais l'adverbe. Le fait d'insérer « do » dans cet énoncé donne davantage de poids à « really ».

53 Finalement même si ces deux cas semblent différents au départ, ils se ressemblent en fait puisque dans les deux occurrences, l'insertion de l'auxiliare «do» modifie a un effet sur le sémantisme de l'adverbe : en 10 il en modifie le sémantisme, et en 11 il le renforce sémantiquement.

\section{Conclusions et perspectives}

L'étude présentée ici a permis de confirmer les résultats d'une étude précédente (Herment-Dujardin, 2008) sur de l'anglais oral spontané également, mais nous avons cette fois pu analyser des «do» non accentués. Les résultats de cette étude nous permettent de mieux faire le lien avec certains éléments prosodiques et sémantiques importants et de répondre à notre question de départ sur les liens entre prosodie et syntaxe. 


\section{Nous pouvons donc affirmer que}

- lorsque « do » remet en cause la réalité du lien sujet/prédicat, l'auxiliaire est alors fortement marqué prosodiquement : il est porteur d'un mouvement mélodique très ample et est noyau de l'unité intonative. Il possède les mêmes caractéristiques prosodiques que d'autres éléments contrastifs, et en particulier la négation. Il est dans ce cas réellement emphatique et possède les traits prosodiques de l'emphase contrastive. Son appellation « do » emphatique est alors totalement justifiée.

- lorsque « do » renforce le lien prédicatif, il est alors nettement moins marqué prosodiquement. Il est tonique de tête de l'unité intonative et non plus noyau. Il possède les mêmes caractéristiques prosodiques que d'autres éléments jouant le même rôle, sans intervention de la syntaxe. Il est encore emphatique, mais il s'agit là d'une emphase non contrastive, donc moins marquée prosodiquement. Syntaxiquement, le marquage reste le même.

- lorsque « do » renforce ou modifie le sens de l'adverbe qui le précède, il est non accentué. C'est l'adverbe qui le précède qui prend l'accentuation, soit de tête, soit nucléaire. « Do » n'est alors plus emphatique du tout. souhaite. discours. communs.

Nous avons donc vu que le même effet syntaxique, l'insertion de l'auxiliaire en contexte affirmatif, ne s'accompagne pas toujours de la même prosodie.

Pour ce qui est du « do » qui renforce un adverbe, on pourrait penser que cela est dû à une contrainte prosodique qui fait qu'on ne peut pas avoir deux accents nucléaires dans une même unité intonative par exemple, ou deux toniques de tête. Donc comme l'adverbe précède l'auxiliaire, c'est lui qui reçoit l'accent. Mais nous pensons que là n'est pas la raison pour laquelle « do » est inaccentué dans ces cas-là.

8 Nous nous demandions en début d'étude si la syntaxe suffisait à elle seule à la mise en relief ou bien si elle était nécessairement accompagnée d'une prosodie marquée. Le présent travail nous permet de répondre que la prosodie vient se surajouter à la syntaxe et en est absolument complémentaire. La prosodie et la syntaxe jouent toutes deux des rôles importants, mais à des niveaux différents. C'est néanmoins l'association entre ces deux niveaux qui nous permet d'accéder à tous les effets de sens que l'on

L'étude du «do» communément appelé emphatique (et pour lequel il faudrait désormais trouver une autre appellation puisque nous avons vu qu'il n'est pas toujours emphatique justement) nous a permis de montrer à quel point la prosodie et la syntaxe sont complémentaires et quel rôle elles jouent sur les subtilités sémantiques du

Une autre étude (Herment, à paraître) sur l'intonation des clivées en «it " semble arriver aux mêmes conclusions. Il serait intéressant de se pencher sur plusieurs structures de focalisation et de voir si l'on pourrait arriver à des traits prosodiques

1 Nous sommes persuadée que les traits prosodiques de l'emphase, contrastive et non contrastive, entretiennent des rapports étroits avec les différents schémas prosodiques de beaucoup de structures de focalisation syntaxique. 


\section{BIBLIOGRAPHIE}

Auran, C., Bouzon, C. \& Hirst, D.J., 2004, The Aix-Marsec Project : an evolutive database of spoken British English, in Proceeding of the second International on Speech Prosody, Nara, March 2004, 561-564.

Boersma, P. \& Weenik, D., 1996, PRAAT : a system for doing phonetics by computer, Report of the Institute of Phonetic Sciences of the University of Amsterdam, 132.

Herment, S., à paraître, Interdépendances entre prosodie et syntaxe dans les clivées en " it ", Actes du XIV colloque de Villetaneuse sur l'anglais oral, 3-4 avril 2008.

Herment-Dujardin, S., 2008, Emphase et prédication : le rôle de la prosodie, in Merle J-M (ed.), La prédication, collection Faits de Langues, vol. 31-32 : 243-252 ;

Herment-Dujardin, S., 2001, L'emphase dans le discours spontané anglais : corrélats acoustiques et prosodiques, Thèse de $3^{\text {ème }}$ cycle, Aix-en-Provence : Laboratoire Parole et Langage.

Hirst, D.J. \& Di Cristo, A., 1998, Intonation Systems : a Survey of twenty languages, Cambridge : CUP. Lapaire, J-M \& Rotgé, W., 1991, Linguistique et Grammaire de l'Anglais, Toulouse : PUM

Roach, P., Knowles, G., Varadi, T., Arnfield, S., 1993, MARSEC : A Machine-Readable Spoken English Corpus, Journal of the International Phonetic Association, 23/2 : 47-53.

Roach, P., 1994, Conversion between prosodic transcription systems : « Standard brotish » and ToBI, Speech Communication 15, 91-99.

\section{NOTES}

1. Pour davatange de détails sur l'alphabet SAMPA : http://www.phon.ucl.ac.uk/home/sampa/xsampa.htm

\section{RÉSUMÉS}

À travers une étude de cas, celui de "do" en anglais, nous nous intéressons aux relations qu'entretiennent la prosodie et la syntaxe dans la parole naturelle. Nous étudions le «do» communément appelé emphatique, i.e. inséré dans l'énoncé alors qu'il n'est pas nécessaire syntaxiquement, dans un corpus de parole naturelle (Aix-Marsec) qui représente 5h30 de parole. Une analyse linguistique est tout d'abord menée et trois "do" sont distingués en fonction de leur rôle au niveau de la relation prédicative; des mesures acoustiques et prosodiques sont ensuite prises: durée de la consonne initiale, hauteur mélodique, mouvement mélodique et ampleur du mouvement sur le mot. Cette étude nous permet d'affirmer que le « do emphatique » n'est prosodiquement pas réalisé de la même façon selon qu'il renforce la relation prédicative ou qu'il nie la relation prédicative donnée avant. Nous montrons que certains "do emphatiques » sont aussi complètement inaccentués. Nous concluons que la prosodie et la syntaxe jouent toutes 
deux des rôles importants pour l'emphase et la focalisation, mais à des niveaux différents : elles sont complémentaires et c'est l'association entre ces deux niveaux qui nous permet de jouer sur les subtilités sémantiques du discours.

The present study focuses on the links between prosody and syntax in a corpus of natural speech through the analysis of "emphatic do", i.e. the auxiliary "do" inserted in an affirmative context. The occurrences of "emphatic do" in the Aix-Marsec corpus ( $5 \mathrm{~h} 30$ of natural speech) are analysed first linguistically and then acoustically and prosodically. Three different linguistic categories are distinguished, depending on the role of "do" at the level of the predicative relation, and acoustic and prosodic measures are taken: length of the initial consonant, pitch, tone. The prosodic realisation of "emphatic do" is shown to be different according to whether "do" reinforces or denies the predicative relation given before. It is also shown that a few occurrences of "do" are totally unaccented. We conclude that prosody and syntax both play important roles for emphasis and focalisation but at different levels: they are revealed complementary and the association between those two levels enables the speaker to play on the semantic subtleties of discourse.

INDEX

Keywords : emphatic do, natural speech, predicative relation, syntax, prosody

Mots-clés : do emphatique, parole naturelle, relation prédicative, syntaxe, prosodie

\section{AUTEUR}

\section{SOPHIE HERMENT}

Laboratoire Parole et Langage, CNRS UMR 6057, Université de Provence 